\title{
DISTANCE LEARNING IS NOT AN ONLINE FACE-TO-FACE - OBSERVATIONS IN MICROELECTRONIC EDUCATION
}

\author{
O. Bonnaud 1,2, L. Fesquet ${ }^{3,4}$ \\ 1 University of Rennes 1, IETR, Rennes, France \\ ${ }^{2}$ GIP-CNFM, Grenoble France \\ ${ }^{3}$ TIMA, Grenoble INP, Grenoble France \\ ${ }^{4}$ CIME Nanotech, University Grenoble Alpes, Grenoble France
}

\begin{abstract}
Due to the pandemic in early 2020, the French government, like many others, decided to temporarily close the universities and asked the faculties to entirely focus on online education or e-learning. In a world where digital contents exponentially increase, this pandemic has been an opportunity to develop distance e-learning. Many politicians found a unique opportunity to decrease or even eliminate face-toface teaching activities. In engineering, this experience has shown clear limits and weaknesses. This paper highlights several problems that raised in microelectronic education and good reasons to move back to face-to-face learning especially for the acquisition of know-how. Indeed, know-how is mandatory for engineers, masters and PhDs in science and technology and this will never be replaced by a virtual experience. Real practice is the necessary price to pay for meeting the technological challenges of the upcoming decade.
\end{abstract}

Keywords: Distance learning during Covid crisis, microelectronics, knowledge and know-how in engineering, digital challenges, virtual teaching limitations.

\section{INTRODUCTION}

Due to the pandemic in early 2020, the French government, like many others, decided to temporarily close the universities and asked the professors to entirely focus on online teaching and e-learning. While the online tools are useful and suitable for students temporarily away from their universities or living in countries with less educational opportunities, the limitations of these tools need to be analysed in depth. Online access to scientific publications, presentations or videos are interesting supports and useful complements to training, especially in the field of microelectronic engineering, which is the expertise field of the authors. Completely changing the educational paradigm from face-to-face to fully online teaching is another matter. This clearly leads to severe disappointments and disastrous results on the know-how acquisition, which is a strong requirement in engineering and, of course, in the field of microelectronics. This paper briefly presents the context of microelectronics, as well as the implementation of distance learning, which has been applied for several months. Due to this quite long experience, several key points are described and highlighted. The article concludes with an evaluation directly carried out by the students after the return of face-to-face classes and labworks on the platforms of the French national network for microelectronic education ("Coordination Nationale pour la Formation en Microélectronique").

\section{MICROELECTRONICS: A HIGH PRIORITY ENGINEERING FIELD}

The paper is set in the context of the higher education in microelectronics. This field is today a national priority due to the very dynamic microelectronic market and the strong needs of the semiconductor companies in terms of technologists and designers. The incredible multiplication of smart connected objects, deployed in the framework of the loT (Internet of Things), has induced many developments in microelectronic circuits and systems covering a wide spectrum of applications, such as communication, health, security, energy, etc. [1]. At the same time, micro-sensors and -actuators are manufactured thanks to microelectronic technologies [2] and highly contribute to the exponential growth of the connected objects [3-4]. As a result, the energy consumption of the internet is also exponentially increasing, roughly by a factor of 2 every 4 years [5]. Figure 1 shows this fast-paced evolution. Without any change, the electrical energy produced in the world will be entirely devoted to the Internet in 2030 . This is of course not desirable. 


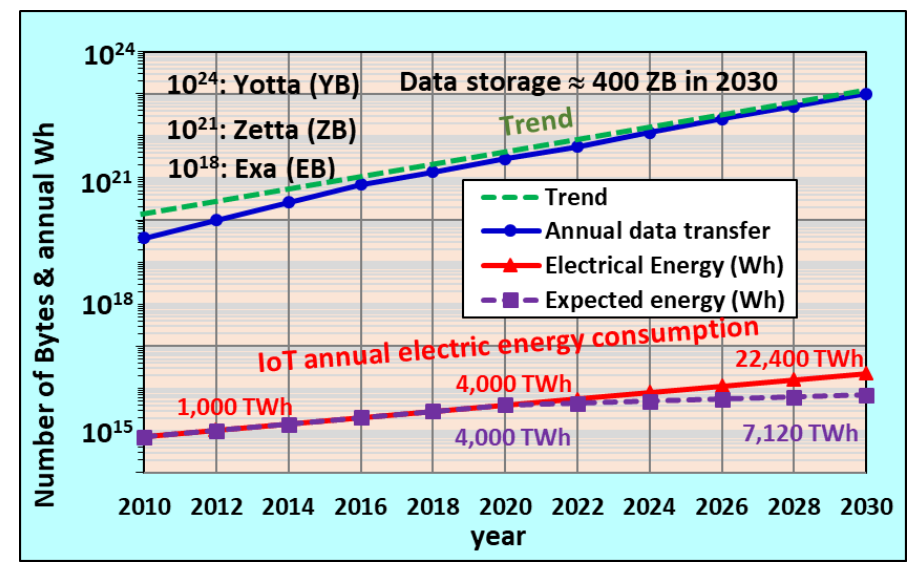

Figure 1. Evolution of the number of bytes transferred in the world and the related electrical energy consumption. Most of this energy is dissipated in electronic circuits and systems. The energy growth must be limited by improving the performance of electronics.

A part of the solution consists in reducing the electrical energy consumption of the electronic devices, circuits and systems by at least one order of magnitude over the next 20 years [6]. The violet dashed curve on Figure 1 shows the energy evolution in that case; it seems negligible on the plot, but notice that the scale is logarithmic. The total energy save annually reaches $14,000 \mathrm{TWh}$. In other words, this corresponds to 14 times the energy of the global air traffic in 2019! Many governments have recognized this challenge and declared the electronic sector as a priority in their strategy.

Today, one of the main difficulties is the shortage of skilled workers in electronics and microelectronics [7]. The solution consists in improving the training of technicians, masters, engineers and PhD students in order to provide them skills and know-how. This is the main motivation of the French national network, which provides a true know-how in microelectronics and nanotechnologies through practice thanks to shared and dedicated platforms at the national level [8].

\section{SWITHCHING TO DISTANCE LEARNING}

In order to improve the learning efficacy, several online tools have been devised to train the students. These are the so-called MOOCs [9] and their associated tools [10]. It also exists documents posted on the university digital platforms such as MOODLE, a learning management system [11] and, more generally, the available online documents such as those found on the "Wikipedia" website, a free online encyclopedia [12]. Notice that these online tools are a good add-on to face-to-face education [13]. Indeed, this gives to the students the ability to retrieve and sort information on the internet such as scientific articles. Moreover, the MOOCs offer a learning process, which goes from the knowledge acquisition to a self-evaluation, quickly giving to the students a feedback on their understanding.

Due to the pandemic and to the emergency situation, the learning activities have been modified in depth to comply with the governmental recommendations and rules, as shown in Figure 2.

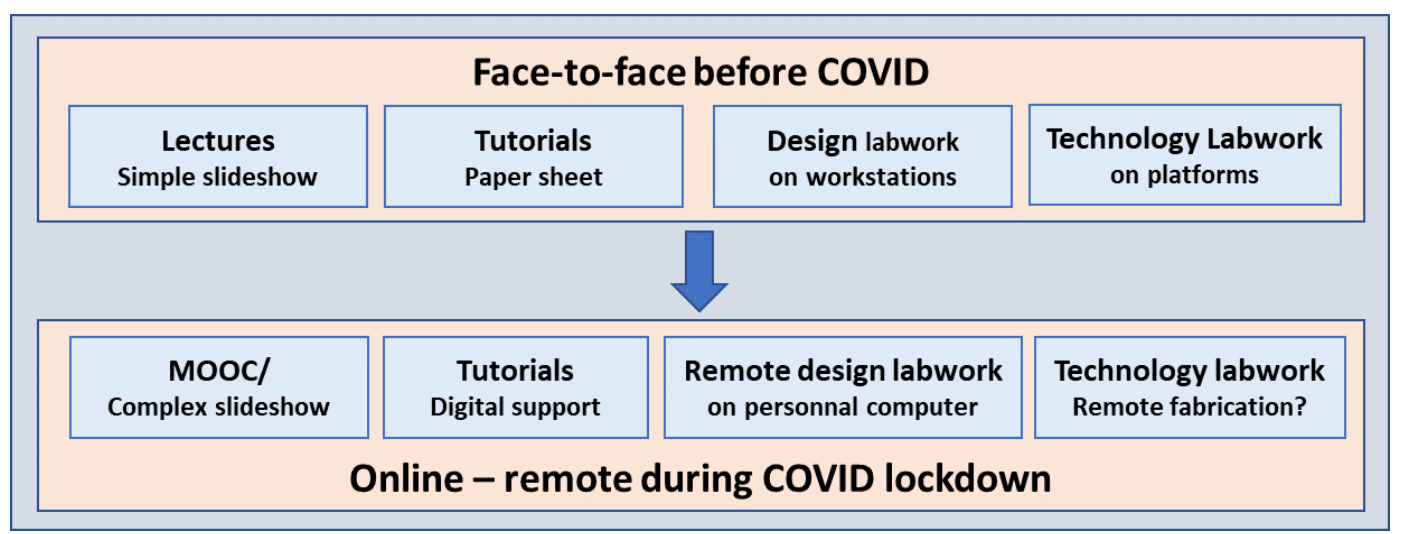

Figure 2. Evolution of learning methods before and after the COVID pandemic. Almost all educational activities shifted to virtual. 
This figure shows the different education activities - including, lectures, tutorials, and labworks in microelectronics for designers and technologists - which has been impacted during the sanitary crisis. Most of these activities have been adapted to be operated in a remote way. This movement has been quickly done with many issues. Nevertheless, the academic community reacted in its majority by first reusing the existing online courses and dedicated tools. Then academics developed dedicated contents in order to facilitate distance learning. This quick shift from face-to-face to remote learning can be divided in four points:

- Reuse of MOOCs (Massive Open Online Courses),

- Adaption of the contents and, sometimes, creation of dedicated documents for online courses,

- Organization of online tutorials,

- Online labwork practice (when it was possible).

In the sequel, we detail each point and describe their strengths and weaknesses.

\subsection{MOOCs}

The first author of this article has already experienced the creation of a MOOC dedicated to the learning of microelectronic technologies and entitled "Integrated Microelectronics Tutorial" [9]. This MOOC is useful for preparing students to the cleanroom labworks of the French national network, GIP-CNFM [1415], dedicated to higher education in microelectronics and nanotechnologies. This MOOC was created within the framework of the European association EAEEIE [16], which was selected to manage successive European projects dedicated to the improvement of higher education in electrical and information engineering [17-18]. With the help of students in the frame of a European mobility, this MOOC dedicated to microelectronics has been translated into several languages (Romanian, Portuguese, German, Finnish, English). This was not only a huge work but we faced issues such as the English version was not already completed before the first release of the initial French version! This implied new investment, which were not anticipated. Moreover, after 6 years, the software has so much evolved that the initial version was no longer available. In conclusion, MOOCs are only applicable if a permanent team is dedicated to their support and maintenance. This assumes that the institutions are able to lock a budget over a long period with the associated human resources. Recently, several academic institutions have created local pedagogical units in order to digitalize learning. Although there is a massive development of digital environments for the teachers and the students (Digital Working Environment), the creation and maintenance of such tools do not appear as an important priority. For instance, the European program called Horizon 2020 -EDUC-SHARE [19] does not mention this action in its eight priorities.

\subsection{Content adaption for online courses}

Face-to-face course are usually supported by a slideshow pointing out in a synthetic manner the main scientific or technical messages. The explanations and methodologies are given by the teacher, which come in complement to the slides. This approach offers a lively progression with frequent interactions with the students. Doing the same remotely through a virtual presentation (videoconference) deeply modifies the student and teacher behavior. Indeed, the students are more passive and reduce their interactions with the professors, while the teachers are frustrated to not see the audience reaction. This makes really difficult the little live adjustments done during the face-to-face lectures. Therefore, the slide content has to be much more developed and the teaching speed reduced in order to maintain minimal exchanges with the students during the videoconferences. As the slides tend to be often overloaded, the core content is much more difficult to catch by the audience. Another consequence is an extra preparation time for the teachers, which is unfortunately not rewarded by a better understanding and assimilation of the fundamentals by the students.

\subsection{Organization of online tutorials}

The tutorial sessions are a good way to assimilate the course fundamentals based on pedagogical examples. They allow students to acquire the concepts, to perform calculations and specially to master the methods and know-how. During these face-to-face sessions, the students lead the action with the support of the teacher who follows them and puts them back on track in case of mistakes. During these sessions, the students often exchange and provide a collective effort improving their understanding and promoting comments and questions, which are essential to develop the innovation spirit. 
The online tutorials are often organized as homework, and the students usually receive their exercises before the session to save time and allow a short preparation. We are far from the panacea because the students are not supported during the tutorial preparation and most of them remain unable to start the exercises. This is clearly a strong limitation for such a practice. Moreover, the innate discussions and exchanges between students and teachers are usually lost by videoconferencing. In conclusion, the spirit of curiosity is lost even if some of the students prepare questions. The physical exchanges are crucial for the communication and, in remote situation, we have observed a wait-and-see attitude conducting students to simply retrieve the results provided by the teacher. All the dynamism of face-toface learning and the associated proactivity has disappeared.

\section{4 - Online labwork practice}

The training of technicians, engineers and doctors in a highly technical field requires the acquisition of knowledge and know-how. This latter is a major part of the skills and qualities for working in the industry and especially in microelectronics [20]. In spite of incredible CAD tools for modeling and designing, microelectronic circuits and systems are so complex that companies always fabricate a physical prototype allowing the product validation and the adjustment of a huge number of parameters Therefore, specialist training requires extensive practical experience, which can only be obtained through laboratory work, projects, and supervised internships [21]. Switching from practical to virtual training leads to major issues..

The first problem is that many experiments cannot be remotely conducted. Indeed, the technical platforms are not intended to perform distance learning. Most of the testbenches, physical devices, circuits and instruments are not designed to be access through the internet. Only few experiments requiring a local operator can be remotely performed. This requires a perfect schedule and a careful preparation by the students' supervisors. This is for instance the case for the unique national circuit test system, a very expensive equipment located in Montpellier and remotely accessible by the French community [22]. Nevertheless, this assumes that the students have already conducted several experiments on physical benches.

Circuit design seems a more favorable activity to be taught online. Indeed, remotely using dedicated software should not be a big issue. Nevertheless, this is not at all the case for several reasons:

- The first difficulty for the students is their ability to access remotely the microelectronic CAD platforms. Indeed, as these CAD tools are quite expensive, their location must remain at the University in order to avoid piracy. This implies to have a connection via a VPN and to install some software tools for redirecting the displays. In practice, many students are not enough familiar with Linux and such tools provoking dissatisfaction and time loss.

- The second issue is for the system administrators and the teachers that have to deliver instructions for remote connections. This task is not obvious because the personal setup of the student computers differs a lot. Indeed, some students own a PC under Linux, others under Windows and, finally, some others under Mac OS. Of course, the number of configurations is not limited to these three situations because the OS versions and releases have to be considered. This is clearly not a cakewalk for managing so many different configurations.

- Another problem is the complexity of microelectronic CAD tools, which deal with huge data libraries (typically several gigabytes) and generate a lot of files. This is particularly disturbing for an isolated student working at home. Indeed, discussing with his neighbor or asking a professor is not so easy even a videoconference is running. In this situation, the first student reaction is to understand by themselves what really happens (which is perfectly healthy), but, as the volume of data and the amount of files are large, it is particularly difficult (for a student) to sort the information.

- In addition, to these hurdles, getting started with microelectronic CAD tools requires to proceed several rigorous steps that a novice user may find very difficult. Indeed, design flows contain a lot of traps for the beginners and require an advanced understanding of the circuit and device models. The role of the teacher is then crucial for organizing the work on the computer, analyzing the physics or the code semantics when the design is made with a HDL (Hardware Design Language). A teacher is usually able to quickly guide the students in laboratory room, by responding to the questions and, more importantly, anticipating the difficulties thanks to a glance on the student group. The online teacher does not have a clear overview of the group 
and its difficulties. This makes delicate the student support and generates a considerable waste of time.

\section{ONE YEAR OF DISTANCE LEARNING}

Since March 2020, in France, the majority of theoretical courses, tutorials and even practical work at the university have been organized remotely by videoconference. Our study is based on observations made in microelectronic education but is surely applicable to other technological disciplines. Since the beginning of 2021 in engineering schools and master degrees, practical activities have been allowed. Thus, the students are back in the classroom for labworks and projects.

Benefiting from these face-to-face situations, a feedback from the students and their teachers has become possible. In the sequel, the proposed analysis mainly concerns engineer and master students in microelectronics, who are usually using of the French national network platforms. On these platforms, they learn the know-how for manufacturing microelectronic circuits, characterizing physical and electrical devices, designing integrated circuits or prototyping embedded systems. This leads to several remarks and comments.

\section{On the remote activities:}

- Being 8 hours per day in front of a computer screen with almost no time-out, isolated at home or in a small flat, is not ideal to keep the attention and, worst, favors a quick loss of focus.

- The students do not (or very marginally) interact with the teacher, as they do not feel his presence. Moreover, online asking questions is more difficult because most of the interactions with the teacher are lost, especially the gesture and body language.

\section{On the pedagogy:}

- Methodologies and scientific approaches require stimulating the students while remote learning favours their passivity.

- Understanding by the students is poor. They are not enough involved because they received a digital document. If they looked at the document before the lab, online labwork is less exciting and considered less difficult thanks to a larger support of the teacher team. Of course, this is just an illusion!

- During the tutorial sessions, most of the students are waiting for the solution instead of seeking by themselves. This implies at the end a reduced autonomy and a lack of questions, which are however essential for the group dynamic.

\section{On the role of the teacher:}

- Teachers are spending a lot more time for preparing because everything should be written down as much as possible. This tends to lower the comments, which are useful for helping the understanding. In face-to-face situations, the teachers naturally limit the pre-written documents and displayed slides. This favors the curiosity and the discussions, for instance by giving analogies that are really useful in engineering or physics.

- In circuit design, labworks can be done online thanks to an important preparatory work for giving access to a large number of students while maintaining the network security. This implies a permanent and efficient technical team for managing the CAD tools and the local servers.

\section{On the internet access:}

- The excessive use of Internet on undersized networks leads to frequent communication breakdowns causing a loss of time at the level of the teacher, but also on the student side. Numerous sessions were cancelled due to an improper national data transfer; this limitation was available for online teaching of high schools as well!

- Increasing the capacity of the servers and connections will solve the problem at the price of an additional work of the technical team, an increase of investment and a higher power consumption, which is not neutral for the carbon footprint. 


\section{CONCLUSIONS}

After the resumption of a few face-to-face sessions in 2021, it appears a few salient points. Firstly, the permanent exchanges during the lab activities by the students with their classmates and teachers make their progression ten times faster than in a remote fashion. Secondly, face-to-face education tends to homogenize the student class and to reduce disparities. Therefore, the progress is collective and the know-how acquisition is much more efficient.

Finally, a survey of several groups of microelectronic engineering students showed that $90 \%$ of students confirm the low effectiveness of online training and report realizing the benefits of practical work on platforms with a teacher. Figure 3 summarizes all these points: the limitations of remote practices and the student feeling and analysis after the resumption of in-person activities. In engineering, permanent remote activities are clearly counter-productive!

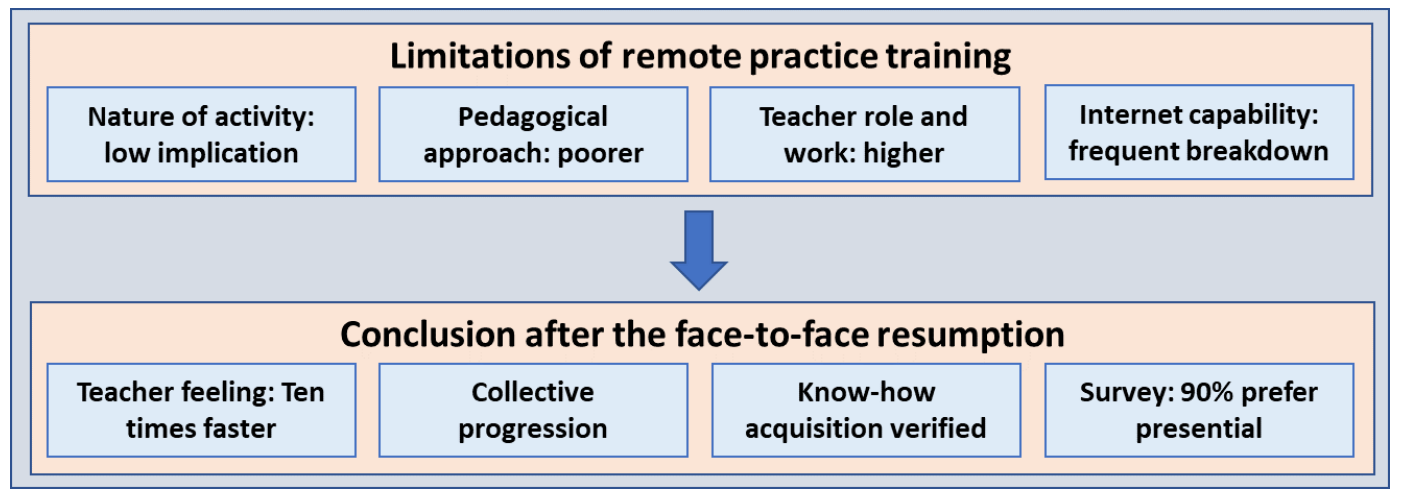

Figure 3: Limitations of the remote practice training and conclusion after resumption of few face-toface practical trainings.

\section{ACKNOWLEDGEMENTS}

The authors want to thank Lorraine Chagoya-Garzon, executive secretary of GIP-CNFM for her fruitful advice for the redaction of this paper.

\section{REFERENCES}

[1] O. Bonnaud, "New Vision in Microelectronics Education: Smart e-Learning and Know-how, a Complementary Approach". ( ) Springer International Publishing AG, part of Springer Nature 2019 V. Uskov et al. (Eds.): KES-SEEL-18 2018, SIST 99, pp. 267-275, 2019.

[2] O. Bonnaud, "New Approach for Sensors and Connecting Objects Involving Microelectronic Multidisciplinarity for a Wide Spectrum of Applications", Int. J. Plasma Environ. Sci. Technol., vol. 10, no. 2, pp. 115-120, 2016.

[3] J Bryzek, "Roadmap for the Trillion Sensor Universe", iNEMI Spring Member Meeting and Webinar, Berkeley, CA, April 2, 2013.

[4] O. Bonnaud, "The technological challenges of microelectronics for the next generations of connected sensors”, Int. J. Plasma Environ. Sci. Technol. vol. 14, pp. 1-8, 2020.

[5] O. Bonnaud, Innovative Strategy to Meet the Challenges of the Future Digital Society, Adv. technol. innov., vol. 6, no. 2, pp. 106-116, 2021.

[6] O. Bonnaud, "Integration, communication and energy consumption: the challenges of higher education in electronics and microelectronics", Proc. of EAEEIE'2019, Ruse (Bulgaria), 4-6 Sept. 2019, IEEExplore, 10.1109/ EAEEIE46886.2019.9000450, pp. 1-4, 17 Feb. 2020.

[7] O. Bonnaud, L. Fesquet, A. Bsiesy, "Skilled manpower shortage in microelectronics: a challenge for the French education microelectronics network," 18th International Conference on Information Technology Based Higher Education and Training (ITHET), doi: 10.1109/ITHET46829.2019.8937384., pp. 1-5, 2019. 
[8] O. Bonnaud, L. Fesquet, "The new strategy based on Innovative Projects in Microelectronics and Nanotechnologies, Invited communication", ECS Journal of Solid State Science and Technology, vol. 2, no. 11, pp. 1-7, 2013.

[9] O. Bonnaud, Tutorial on Microelectronics Technology (2002), Accessed May 2021, Retrieved from https://microelectronique.univ-rennes1.fr/en/index.html

[10] A. Fox, "From MOOCs to SPOCs", in Proc. of ACM, vol. 56, no. 12, pp. 38-40, 2013.

[11] Moodle definition, Accessed May 2021: Retrieved from https://moodle.org/?lang=en

[12] Wikipedia, The Free Encyclopedia, Accessed May 2021, Retrieved from https://en.wikipedia.org/wiki/English_Wikipedia

[13] O. Bonnaud and L. Fesquet, MOOC and the Practice in electrical and information engineering: complementary approaches. In Proc. of ITHET'2016, Istanbul (Turkey), pp. 1-4, 2016.

[14] Microelectronics and French Nanotechnologies Training Network. GIP-CNFM: "Coordination Nationale pour la formation en Microélectronique and nanotechnologies", Accessed 2021/05/04, Retrieved from http://www.cnfm.fr

[15] O. Bonnaud, P. Gentil, A. Bsiesy, S. Retailleau, E. Dufour-Gergam, J.M. Dorkel, "GIP-CNFM: a French education network moving from microelectronics to nanotechnologies", In Proc. of EDUCON'11; Amman (Jordan), ISBN978-1-61284-641-5, pp 122-127, 2011.

[16] EAEEIE, European Association for Education in Electrical and information Engineering. Accessed May 2021, Retrieved from http://www.eaeeie.org/

[17] M.J. Martins, J.M. Thiriet et al. "Towards the Harmonization of Electrical and Information Engineering Education in Europe", ISBN 972-97738-2-3, Lisboa-Nancy 2003, 203 pages, Ed. EAEEIE, 2005.

[18] J.M. Thiriet; et al.. "Results and dissemination of the EIE-Surveyor thematic network", EAEEIE Annual Conference, 2009, IEEE Volume Issue June 2009, pp.1-6, 2009.

[19] European program called Horizon 2020, Retrieved from https://euraxess.ec.europa.eu/jobs/hosting/manager-educ-share-european-project

[20] O. Bonnaud and L. Fesquet, "The practice in microelectronics: a mandatory complement of the online courses in the context of digital society". 33rd Symposium on Microelectronics Technology and Devices (SBMicro), doi: 10.1109/SBMicro.2018.8511430, pp. 1-5, 2018.

[21] O. Bonnaud, "Practice-oriented pedagogical strategy of the French microelectronics and nanotechnologies network". In Proc. of WSEAS'2015, Kuala-Lumpur (Malaysia), in Recent Advances in Education and Educational Technology.

[22] L. Latorre, B. Pradarelli, P. Nouet, Integrated Circuits Testing: Remote Access to Test Equipment for Labs and Engineering, Intern. Journal of Online and Biomedical Engineering (iJOE), vol. 5, 2009. 